\title{
Anaesthetic Management of a Case of Pheochromocytoma
}

\author{
Panta $\mathrm{S}^{1}$, Rai $\mathrm{S}^{2}, \mathrm{KC} \mathrm{NB}^{3}$
}

${ }^{1}$ Sunita Panta, Anaesthesiologist, ${ }^{2}$ Shashi Rai, Consultanat Anaesthesiologist, ${ }^{3}$ Nagendra Bahadur KC, Consultant Anaesthesiologist, Shree Birendra Hospital, Chhauni

\begin{abstract}
Pheochromocytoma is rare, accounting for less than $0.1 \%$ of hypertensive population. In this report, we describe a 40-year-old male a diagnosed case of pheochromocytoma who underwent adrenalectomy under general anaesthesia with epidural analgesia. The patient was adequately prepared with alpha adrenergic blockers. Intraoperative course was stormy but was managed with antihypertensives, inotropes and intravenous fluid. The patient was electively ventilated overnight and had an uncomplicated recovery.
\end{abstract}

Pheochromocytoma is a rare medical condition and an anaesthesiologist comes across it only a few times in his or her practice. Therefore there is a limited exposure in management. Furthermore hemodynamic instability encountered intra and postoperatively itself is a challenge. Hence management of a case of pheochromocytoma demands a meticulous preoperative preparation, advanced monitoring devices and good interdepartment coordination preferably in a tertiary medical center for a favourable outcome.

Key words: Haemodynamic instability, inotropes, pheochromocytoma, Sodium Nitroprusside.

\section{Case History}

A 40 year old male weighing $65 \mathrm{~kg}$ was diagnosed 2 months back as a case of pheochromocytoma. He presented with headache, sweating and palpitation along with hypertension. He was on treatment for 1year with tablet prazosin $7.5 \mathrm{mg}$. twice daily, tablet aquazide $25 \mathrm{mg}$ once daily and tablet amlodipine AT once daily. He was now posted for laparotomy and excision of the tumor.

On examination, he was alert, afebrile and cooperative. Pulse rate was 63/minute, regular and good volume. Blood pressure recording over right upper limb was 150/90 mm $\mathrm{Hg}$ in supine and 140/80 mm Hg in standing position. Systemic examination and airway assessment revealed no abnormality.

Investigations revealed a $\mathrm{Hb}-12$ gm \%, FBS$122 \mathrm{mg} / \mathrm{dl}$. Electrocardiogram showed left ventricular Address for correspondence: sunitapanta@gmail.com hypertrophy. Echocardiogram revealed concentric left ventricular hypertrophy with ejection fraction of $60 \%$. MRI showed Lt adrenal mass. Renal CT angiogram revealed Lt adrenal mass and Lt renal artery stenosis. Suspecting pheochromocytoma specific tests were carried out which were positive only in the second time. 24 hour urinary catecholamines $625 \mathrm{mcg} /$ day (normal 0275) VMA-26.53mg\% (normal<13.6) and metanephrines $1.83 \mathrm{mg} /$ day ( normal <1.0).

\section{Anaesthetic Management}

Pre operative preparation with Alpha blocker prazosin, amlodipine and aquazide ensured adequate control of hemodynamic status. Patient was given tablet diazepam $10 \mathrm{mg}$ on the previous night and on morning of the surgery and was kept fasting overnight. Adequate blood was arranged. 
In the operation theater pulse oximeter, ECG and NIBP were connected for monitoring. 16G intravenous cannula was inserted over right forearm and $20 \mathrm{G}$ cannula over left radial artery for invasive monitoring. The patient was then positioned in sitting position and 18G Epidural catheter inserted in T9- T10 space. For confirmation of epidural cannulation $2 \%$ plain xylocaine was injected and adrenaline was avoided. For analgesia $50 \mathrm{mcg}$ of fentanyl in $10 \mathrm{cc}$ normal saline was injected through the epidural catheter. Foley's urinary catheter was inserted after induction. This was followed by cannulation of right subclavian vein for CVP monitoring, fluid and drug administration.

After pre oxygenation, the patient was induced with midazolam $2 \mathrm{mg}$, xylocard $40 \mathrm{mg}$, fentanyl 150 $\mathrm{mcg}$ and propofol $140 \mathrm{mg}$. He was intubated with 7.5 $\mathrm{mm}$ cuffed oral endotracheal tube with rocuronium 50 $\mathrm{mg}$ iv. The blood pressure shot up to $180 / 100 \mathrm{~mm}$ hg which was managed by esmolol $30 \mathrm{mg}$ in divided doses. Anesthesia was maintained with oxygen, vecuronium, fentanyl, midazolam, isoflurane, titrated doses of sodium nitroprusside and epidural bupivacaine in regular intervals. During intra operative handling of tumor there was a sudden surge of blood pressure with tachycardia which was managed by stepping up the dose of SNP, I.V boluses of xylocard and increasing the depth of anesthesia. After ligation of the tumor there was severe refractory hypotension, which was managed initially by colloids and incremental doses of norepinephrine. A very high dose of norepinephrine infusion was required to combat the hypotension.

After the surgery the patient was put on elective ventilation on CMV mode overnight in the post operative ward. Norepinephrine was gradually tapered. Fluctuation in blood glucose levels were managed with insulin and dextrose. Analgesia was maintained via epidural catheter with top up doses of bupivacaine. Patient was extubated next morning. The further course was uneventful. Patient was discharged home days later. The biopsy report confirmed the diagnosis of pheochromocytoma.

\section{Discussion}

In 1886, Fränkel made the first description of a patient with pheochromocytoma however the term was first coined by Ludwig Pick, a pathologist, in 1912. In 1926, Roux (in Switzerland) and Mayo (in U.S.A.) were the first surgeons to remove pheochromocytomas.

A phaeochromocytoma (PCC) or pheochromocytoma, is a neuroendocrine tumor of the medulla of the adrenal glands (originating in the chromaffin cells), or extra-adrenal chromaffin tissue that failed to involute after birth and secretes excessive amounts of catecholamines. Extra-adrenal pheochromocytomas (paragangliomas) are closely related, though less common, tumors that originate in the ganglia of the sympathetic nervous system and are named based upon the primary anatomical site of origin.

\section{Incidence}

Pheochromocytoma is seen in between 2-8 in $1,000,000$ with approximately 1000 cases diagnosed in United States yearly. It mostly occurs in young or middle age adults, though presents earlier in hereditary cases. These tumors can form a pattern with other endocrine gland cancers which is labeled multiple endocrine neoplasia (MEN). Pheochromocytoma may occur in patients with MEN 2 and MEN 3 (MEN 2B).

Inadults, approximately $80 \%$ of pheochromocytomas are unilateral and solitary, $10 \%$ are bilateral, and $10 \%$ are extra-adrenal. The tumors are made up of large, polyhedral, pleomorphic chromaffin cells. Fewer that $10 \%$ of these are malignant. Local invasion of surrounding tissues or distant metastases indicate malignancy.

\section{Clinical Features}

The signs and symptoms of a pheochromocytoma are those of sympathetic nervous system hyperactivity including: skin sensations, flank pain, hypertension,
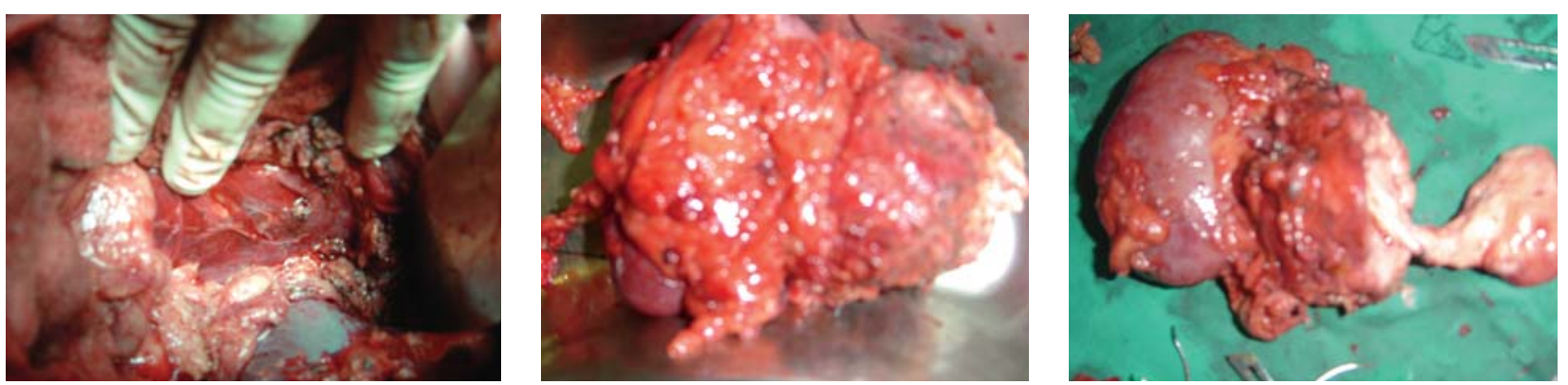

Photograph of Adrenal Mass and Kidney 
palpitations, anxiety diaphoresis, headaches and hyperglycaemia. A pheochromocytoma can also cause resistant hypertension. The most common presentation is paroxysms of headache, diaphoresis and palpitations.

Patients experiencing symptoms associated with pheochromocytoma should be aware that it is rare. However, it often goes undiagnosed until autopsy therefore patients might wisely choose to take steps to provide a physician with important clues, such as recording whether blood pressure changes significantly during episodes of apparent anxiety.

\section{Diagnosis}

The diagnosis can be established by measuring catecholamines and metanephrines in plasma or through a 24-hour urine collection. Care should be taken to rule out other causes of adrenergic excess like hypoglycemia, stress, exercise, and drugs affecting the catecholamines (methyldopa, dopamine agonists, or ganglion blocking antihypertensives). Various foodstuffs (e.g. vanilla ice cream) can also affect the levels of urinary metanephrine and VMA (vanillylmandelic acid). Imaging by computed tomography or a T2 weighted MRI of the head, neck, and chest, and abdomen can help localize the tumor. Tumors can also be located using lodine-123 meta-iodobenzylguanidine (I123 MIBG) imaging.

\section{Differential Diagnosis}

The differential diagnosis of pheochromocytoma includes: anxiety disorders, paragangliomas essential hypertension, renovascular hypertension and carcinoid syndrome.

\section{Treatment}

Surgical resection of the tumor is the treatment of first choice, either by open laparotomy or else laparoscopy. Given the complexity of perioperative management, and the potential for catastrophic intra and postoperative complications, such surgery should be performed only at centers experienced in the management of this disorder. In addition to the surgical expertise that such centers can provide, they will also have the necessary endocrine and anesthesia resources.

\section{Preoperative preparation}

Either surgical option requires prior treatment with the non-specific and irreversible alpha adrenoceptor blocker (Phenoxybenzamine). Doing so permits the surgery to proceed while minimizing the likelihood of severe intraoperative hypertension. Some authorities would recommend that a combined alpha/beta blocker such as labetalol also be given in order to slow the heart rate. Regardless, a "pure" beta blocker such as atenolol must never be used in the presence of a pheochromocytoma due to the risk of such treatment leading to unopposed alpha agonism and, thus, severe and potentially refractory hypertension.

The patient with pheochromocytoma is invariably volume depleted. In other words, the chronically elevated adrenergic state characteristic of an untreated pheochromocytoma leads to near-total inhibition of reninangiotensin activity, resulting in excessive fluid loss in the urine and thus reduced blood volume. Hence, once the pheochromocytoma has been resected, thereby removing the major source of circulating catecholamines, a situation arises where there is both very low sympathetic activity and volume depletion. This can result in profound hypotension. Therefore, it is usually advised to "salt load" pheochromocytoma patients before their surgery. This may consist of simple interventions such as consumption of high salt food pre-operatively, direct salt replacement or through the administration of intravenous saline solution. The anaesthetic management of a patient with pheochromocytoma requires consideration in following points:

- $\quad$ Preoperative preparation with adrenergic blocking agents.

- Use of an anaesthetic agent which is not associated with release of endogenous catecholamines and does not sensitize the myocardium to high levels of circulating catecholamines.

- $\quad$ Adequate fluid and blood administration, including preoperative transfusion if necessary.

- Careful monitoring during surgery, including direct arterial pressure, central venous pressure, electrocardiogram, urinary output, and blood gas determinations.

- $\quad$ Ready availability of all pharmacological agents appropriate for the control of hypertension, hypotension, and cardiac arrhythmias.

To help assess the adequacy of preoperative management of pheochromocytoma, the following Roizen criteria $^{1}$ should be met in order to reduce perioperative morbidity and mortality:

- $\quad$ No in-hospital blood pressure $>160 / 90 \mathrm{~mm} \mathrm{Hg}$ for 24 hours prior to surgery. 
- No orthostatic hypotension with blood pressure $<80 / 45 \mathrm{~mm} \mathrm{Hg}$.

- $\quad$ No ST or T wave changes for 1 week prior to surgery.

- $\quad$ No more than 5 premature ventricular contractions per minute.

In our case report, the patient was adequately prepared with prazosin and amtas and aquazide and he met all the parameters of Roizen criteria. He was premedicated with diazepam before the surgery. There are reports suggesting the dangers of using drugs like morphine and atracurium which potentially release histamine because of the risk of provoking catecholamine release from chromaffin granules ${ }^{2}$.

\section{Choice of Anaesthesia}

The combination of adequate regional anesthesia with general anesthesia provides satisfactory conditions for the initial surgical incision and exposure of the tumor. We chose to insert the epidural catheter also under local anesthesia before induction. Test dose was given only with $2 \%$ plain lignocaine. Adrenaline was avoided even in test dose for epidural catheter. A segmental blockade was achieved at mid to low thoracic level.

\section{Monitoring}

Peripheral venous, arterial and central venous catheters were placed under local anesthesia and hemodynamic monitoring was established together with ECG and pulse oximeter. However invasive arterial blood pressure monitoring was not possible due to machinery error. Extensive monitoring is required for such surgeries to monitor both the hypertensive crises and the post ligation hypotensive episodes. Manipulation of the tumor, however gently performed, usually causes a brisk hemodynamic response.

\section{Drugs to Combat Haemodynamic Alteration}

Intraoperatively, intravenous phentolamine, nitroglycerin and sodium nitroprusside are most often used to control blood pressure swings. Hull claimed that phentolamine is less satisfactory because tachycardia is an invariable problem ${ }^{3}$. We decided to use SNP to manage the intraoperative hypertension as there was ample evidence that SNP has been successfully used $^{4,5,6}$. The most widely quoted alternative to SNP is phentolamine, a competitive ${ }_{1}$ - and weak ${ }_{2}$-adrenoceptor antagonist, which can be given intravenously as an infusion or as incremental doses of 1-2 mg. Just prior to venous ligation of the tumor, anticipating hypotension, low dose dopamine was started along with volume loading and tapering of SNP.

\section{Postoperative care}

The main postoperative complication of surgery for pheochromocytoma is persistent arterial hypotension which may be refractory to intravascular volume replacement and adrenoceptor agonists. Our patient responded to Dopamine infusion which was later tapered and finally weaned off after 2days. Our patient also developed hypoglycemia during the post operative period and had to be given dextrose infusions. This hypoglycemia is due to the excessive rebound secretion of insulin after the removal of catecholamine secreting pheochromocytoma ${ }^{7,8}$.

\section{Conclusion}

Pheochromocytoma constitutes only a small fraction of hypertensive patients. Many of these cases remain undiagnosed. An anaesthesiologist may encounter these cases only a few times in practice. Therefore even though many of us are aware of the management aspect academically handling such a situation may be very difficult. Preoperative preparation of the patients is of utmost importance.

The management of patients with pheochromocytoma remains a challenge for the anesthesiologist despite the advent of new drugs and techniques. Our role in the successful outcome of such surgeries begins from adequate pre operative preparation, extensive intra operative monitoring and careful follow up during post operative period. Prognosis is usually good if the tumor is detected early to avoid major complications related to catecholamine excess.

\section{References}

1. Roizen MF, Horrigan RW, Koike M, Eger, IE. A prospective randomized trial of four anesthetic techniques for resection of pheochromocytoma. Anesthesiology. 1982; 57: A 43.

2. C. Prys-Roberts. Phaeochromocytoma-recent progress in its management. $\mathrm{Br} \mathrm{J}$ Anaesth 2000; Vol. 85, No. 1: 44-57.

3. Hull CJ. Phaeochromocytoma: diagnosis, pre-operative preparation, and anaesthetic management. Br J Anaesth 1986; 58: 1453-68. 
4. Brown BR Jr. Anaesthesia for phaeochromocytoma. International Practice of Anaesthesia. Oxford: Butterworth Heinemann, 1996; 1/83/1-7.

5. Munro J, Hurlbert BJ, Hill GE. Calcium channel blockade and uncontrolled blood pressure during phaeochromocytoma surgery. Can J Anaesth 1995; 42: 228-30.

6. Tjeuw M, Fong J. Anaesthetic management of a patient with a single ventricle and pheochromocytoma. Anaesth Intens Care 1990; 18: 567-9.
7. G. E. Wilkins, N. Schmidt, and W. A. Doll. Hypoglycemia following excision of pheochromocytoma. Can Med Assoc J. 1977 February 19; 116(4): 367-368.

8. Masako A, Takaya K, Yukio I, Takao O, Yoshihide F. Hypoglycemia Induced by Excessive Rebound Secretion of Insulin after Removal of Pheochromocytoma. World J. Surg. 1990; 14: 317324. 